\title{
A study of genes controlling carcinogenesis in a regenerative model flatworm Macrostomum lignano
}

\author{
Kirill Ustyantsev, Valeriya Vavilova \\ Sector of molecular-genetic \\ mechanisms of regeneration \\ ICG SB RAS \\ Novosibirsk, Russia \\ ustyantsev@bionet.nsc.ru, \\ valeriya-vavilova@bionet.nsc.ru
}

\author{
Mikhail Biryukov \\ Interinstitutional laboratory of \\ molecular \\ paleogenetics and paleogenomics \\ ICG SB RAS \\ Novosibirsk, Russia \\ birykov@bionet.nsc.ru
}

\author{
Eugene Berezikov \\ Sector of molecular-genetic \\ mechanisms of regeneration \\ ICG SB RAS \\ Novosibirsk, Russia \\ European Research Institute \\ for the Biology of Ageing \\ Groningen, The Netherlands \\ ORCID 0000-0002-1145-2884 \\ eberez@bionet.nsc.ru
}

\begin{abstract}
Free-living flatworm Macrostomum lignano is a novel model organism that provides a genetically tractable experimental system to specifically study the interplay between regulation of regeneration and cancer. Here, we tested two chemical carcinogens (MMS and CsA) and short wavelength ultraviolet irradiation (UVC) in M. lignano in order to identify genes and molecular pathways underlying carcinogenic response in this flatworms. For the first time, sensitivity of $M$. lignano to hard UV was evaluated, and it was shown that the worm can easily tolerate sterilization-level doses of higher than $100 \mathrm{~mJ} / \mathrm{cm} 2$. Using differential gene expression analysis based on generated RNA-Seq data, common and individual patterns of $M$. lignano transcriptional response to the induced carcinogenesis by the tested stimuli were determined. This allowed us to select promising candidate genes for functional studies using RNAi knock-down screens and for determination of their role in stem cells regulation under regeneration and homeostasis in M. lignano.
\end{abstract}

Keywords - Macrostomum lignano, flatworms, carcinogenesis, RNA-Seq, ultraviolet

\section{Introduction}

The restriction of regeneration is thought to evolve as a measure against cancer. However, highly regenerative flatworms do not have cancer, suggesting that they evolved some other regulatory mechanism to keep their cells under control while allowing the process of regeneration. Free-living regenerative flatworm Macrostomum lignano is a novel model organism that provides a genetically tractable experimental system to specifically study the interplay between regulation of regeneration and cancer [1]. Here, we studied changes in transcriptional profile in $M$. lignano which occur upon treatment by both chemical and physical carcinogenic factors to identify genes which have the most impact on stem cell regulation and proliferation control in this flatworm.

\section{Methods and Algorithms}

Worms were treated separately by two chemical carcinogenic compounds for 24 h: genotoxic methylmethansulfonate (MMS, $0.5 \mathrm{mM}$ ) and non-genotoxic cyclosporin A (CsA, $0.2 \mu \mathrm{M})$, concentrations of which were determined previously [2]. For a physical carcinogenic factorworms were exposed to short wavelength ultraviolet irradiation (UVC, $254 \mathrm{~nm}, 100 \mathrm{~mJ} / \mathrm{cm} 2$ ) and allowed to recover for $1 \mathrm{~h}$ or $6 \mathrm{~h}$. Each of the treatments, as well as intact controls, were done in four biological replicates. Total RNA was isolated from the samples and used for RNA-Seq library preparation using the Smart-3SEQ protocol [3]. The libraries were sequenced on the Illumina NextSeq500. Raw reads were processed by TrimGalore and mapped to Mlig_3_7 genome assembly using STAR (v.2.5.4b). Differential gene expression (DE) analysis was performed with edgeR [4], setting FDR threshold to 0.001 .

\section{Results}

Sensitivity of $M$. lignano to UVC was studied for the first time. It was determined that the animals can handle UVC doses higher than $100 \mathrm{~mJ} / \mathrm{cm} 2$ (close to the sterilization of viral particles) without noticeable phenotype and lethality. A total of 20 RNA-Seq libraries were obtained, sequenced and analysed in this study. MDS analysis in edgeR supported good separation and clusterization of all the libraries according to the treatments applied with an exception of the CsA-treated samples, suggesting that CsA treatment has limited effect on transcriptional changes. Summary on the DE analysis is shown in Table 1. We assumed that $M$. lignano genes responsible for carcinogenesis regulation should be upregulated upon the treatments. To further narrow the search, we focused only on upregulated DE genes, which are specific to the worm's stem cells (neoblasts) and progenitors (Table 2). Overlapping these lists resulted in the final list of 30 candidate genes, which are being further experimentally investigated.

TABLE 1. SUMMARY OF THE DE ANALYSIS. FDR $<0.001$

\begin{tabular}{|l|l|l|}
\hline Treatment & $\begin{array}{l}\text { Number of } \\
\text { upregulated } \\
\text { genes }\end{array}$ & $\begin{array}{l}\text { Number of } \\
\text { downregulated } \\
\text { genes }\end{array}$ \\
\hline MMS & 1633 & 2560 \\
\hline CsA & 31 & 6 \\
\hline 1h post UVC & 2037 & 1063 \\
\hline 6h post UVC & 3252 & 2048 \\
\hline
\end{tabular}


TABLE 2. NUMBER OF DE GENES UPREGULATED IN M. LIGNANO STEM CELLS

\begin{tabular}{|l|l|l|l|}
\hline Cell type & MMS & 1h post UVC & 6h post UVC \\
\hline Only neoblasts & 108 & 27 & 34 \\
\hline $\begin{array}{l}\text { Neoblasts and } \\
\text { progenitors }\end{array}$ & 306 & 101 & 144 \\
\hline
\end{tabular}

\section{Conclusion}

The obtained data lay the foundation for the development of comparative genomics among Macrostomum species. The list of promising candidate genes for further functional studies of molecular mechanisms underlying carcinogenesis and regeneration control in M. lignano was obtained.

\section{ACKNOWLEDGMENT}

The work was supported by Russian Foundation for Basic Research (RFBR) grant No. 18-04-01011.

\section{REFERENCES}

[1] S. Mouton, J. Wudarski, M. Grudniewska, and E. Berezikov, "The regenerative flatworm macrostomum lignano, a model organism with high experimental potential," Int. J. Dev. Biol., vol. 62, no. 6-8, pp. 551-558, 2018.

[2] M Biryukov, I Sukhikh, V Vavilova, K Ustyantsev, and E Berezikov, "Establishing free-living flatworm Macrostomum lignano as a model to study links between regeneration and cancer," Systems Biology and Bioinformatics, pp. 09-09, Aug. 2018.

[3] J. W. Foley et al., "Gene expression profiling of single cells from archival tissue with laser-capture microdissection and Smart-3SEQ," Genome Res., vol. 29, no. 11, pp. 1816-1825, Nov. 2019.

[4] M. D. Robinson, D. J. McCarthy, and G. K. Smyth, "edgeR: A Bioconductor package for differential expression analysis of digital gene expression data," Bioinformatics, vol. 26, no. 1, pp. 139-140, Nov. 2009. 